\title{
Pankreaskarzinom - Diagnostik
}

\author{
G. A. Pistorius \\ M. K. Schilling
}

\section{Pancreatic cancer - diagnostic}

Das Pankreaskarzinom ist die vierthäufigste tumorassoziierte Todesursache in westlichen Industriestaaten (19). Es ist charakterisiert durch eine überwiegend späte Diagnosestellung, aggressives Wachstumsverhalten und daraus resultierend eine äußerst schlechte Prognose. Die Gesamtmortalität des Pankreaskarzinoms (1-Jahresmortalität bis zu 80\%) ist nahezu identisch mit der Inzidenz.

Pankreaskarzinome sind aufgrund der klinischen Präsentation und Prognose von Papillenkarzinomen zu unterscheiden und lassen sich in fünf histologische Gruppen unterteilen. Dabei stellen die duktalen Karzinome mit 75-90\% die häufigste Tumorentität dar (Rest: Azinuszellkarzinome, gemischte, unbestimmte, unklassifizierte Zelltypen).

Durch Senkung der chirurgischen Mortalität und Morbidität konnte in Abhängigkeit vom Tumorstadium bei entsprechender Selektion die 5-Jahresüberlebensrate in den letzten Jahren in Schwerpunktzentren auf 20 bis über $30 \%$ angehoben werden $(1,5,26)$.

\section{Epidemiologie, Ätiologie und Risikofaktoren}

Die Inzidenz des Pankreaskarzinoms beträgt ca. $5-10$ pro 100000 pro Jahr. Das Risiko Männer/Frauen beträgt 2:1 mit einem Häufigkeitsgipfel zwischen dem 6. und 8. Lebensjahrzehnt. Dem Tabakkonsum und der Exposition gegenüber aromatischen Aminen kommt unter den exogenen Einflüssen die stärkste Bedeutung zu (relatives Risiko 2-3) $(6,8,14,25)$. Als weitere Risikofaktoren werden chronische Pankreatitiden (relatives Risiko ca. 15) (17) und Alkoholkonsum (22) angeschuldigt. Darüber hinaus gehen die autosomal-dominant vererbten here- ditären Pankreatitiden mit einem erhöhten Risiko einher. An genetischen Alterationen findet sich in Pankreaskarzinomen eine Aktivierung des dominant transformierenden Onkogens Ki-ras (ca. 90\%) sowie eine Inaktivierung verschiedener Tumorsuppressorgene (P 53, $\mathrm{DPC}_{4}, \mathrm{CDKN}_{2}, \mathrm{BRCA}-2$ ).

kurzgefasst: Der Häufigkeitsgipfel des Pankreaskarzinoms liegt zwischen dem 6 . und 8. Lebensjahrzehnt. Tabak-, Alkoholkonsum, rezidivierende Pankreatitiden und genetische Alterationen sind nachgewiesene Risikofaktoren.

\section{Diagnostik}

\section{Klinische Symptomatik}

Ca. 75\% der Pankreaskarzinome sind im Caput lokalisiert, 20\% im Corpus und 5\% im Caudabereich und führen im Gegensatz zu den papillennahen Tumoren häufig zu uncharakteristischen Symptomen wie Oberbauchbeschwerden, Gewichtsverlust, Rückenschmerzen, spezifischer zu schmerzlosem Ikterus, neu aufgetretenem Diabetes mellitus oder Pankreatitis (13). Insbesondere bei Patienten $>60$ Jahre mit neu aufgetretenen Rückenbeschwerden oder Diabetes mellitus sollte immer an ein Pankreaskarzinom mitgedacht werden, um Verzögerungen in der Diagnostik zu vermeiden.

Da die klinische Untersuchung häufig unergiebig ist, stehen die bildgebenden Verfahren im Mittelpunkt - sie folgen sinnvollerweise einem Stufenkonzept (Abb 1).

Institut

Abteilung für Allgemein-, Viszeral- und Gefäßchirurgie, Universitätskliniken des Saarlandes, Homburg/Saar

Korrespondenz

Prof. Dr. med. Martin K. Schilling · Abteilung für Allgemein-, Viszeral- und Gefäßchirurgie

Universitätskliniken des Saarlandes · 66424 Homburg/Saar · Tel.: 06841/162 2605 · Fax: 06841/162 2697 .

E-Mail: martin.schilling@uniklinik-saarland.de

eingereicht: 7.6 .2002 - alkzeptiert: 9.9.2002

Bibliografie

Dtsch Med Wochenschr 2002;127:2326-2329 • @ Georg Thieme Verlag Stuttgart · New York · ISSN 0012-0472 


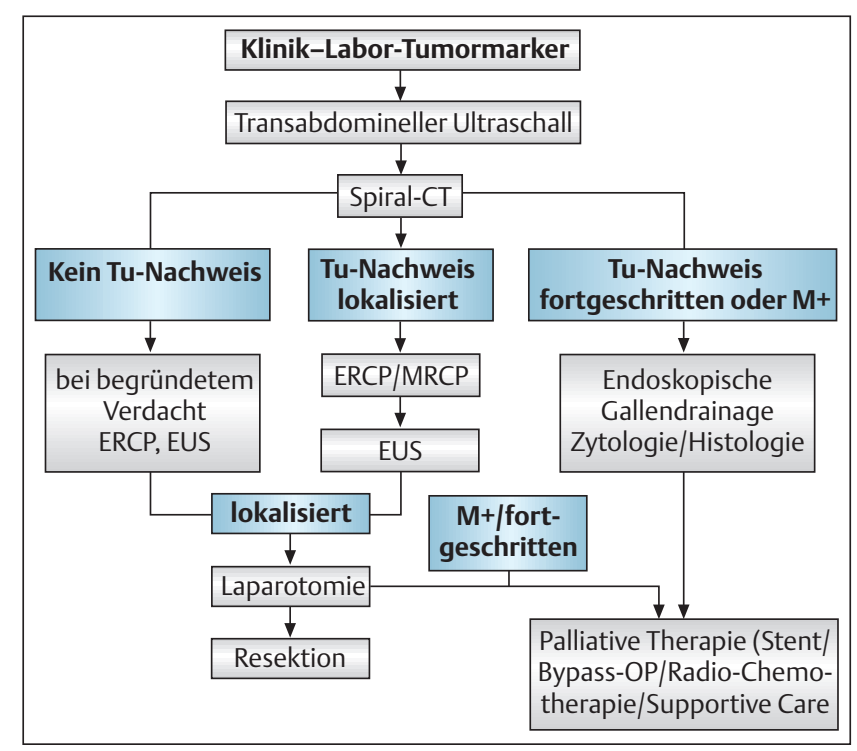

Abb.1 Stufenkonzept der Diagnostik des Pankreaskarzinoms. (M+ = Metastasierung).

\section{Transabdomineller Ultraschall (US)}

Aufgrund der kostengünstigen Verfügbarkeit stellt der US heute die erste und wichtigste Untersuchung bei Verdacht auf eine Pankreasraumforderung dar (13). Die Beurteilung, insbesondere der Caudaregion, ist durch retroperitoneale Lage des Organs und häufige Luftüberlagerungen erschwert. Es gilt, dass jede hypodense Struktur im Pankreas karzinomverdächtig ist und die Sensitivität ab einer Tumorgröße von $>1 \mathrm{~cm}$ sprunghaft ansteigt (bis $72 \%$ ). Dabei wird sonographisch die Tumorausdehnung häufig unterschätzt $(20,23)$. Ein wichtiger Vorteil der US liegt in der hohen Sensitivität für die Detektion von Lebermetastasen (3).

\section{Farbduplexsonographie}

Durch die Duplexsonographie lassen sich direkte und indirekte Hinweise auf eine Gefäßinvasion erfassen, unter Umständen lassen sich pathologische Tumorrandgefäße als zusätzliches Malignitätskriterium darstellen (23).

kurzgefasst: Die sonographische Diagnostik ist insbesondere in der Caudaregion erschwert, die Tumorgröße wird in der Regel unterschätzt. Die Duplexsonographie dient dem Nachweis einer Gefäßinfiltration.

\section{Computertomographie}

Die Computertomographie (ggf als Spiral-CT, Dünnschicht-CT oder Angio-CT) ist nach wie vor das Standardverfahren der bildgebende Diagnostik beim Pankreaskarzinom mit einer Sensitivität von ca. 90\%. Problem der CT-Diagnostik ist die Vorhersage der Resektabilität, welche lediglich bei 60-70\% liegt und auf der Unschärfe des Verfahrens bei fraglicher Gefäßinfiltration, Infiltration ins Mesenterium und nach retropankreatisch beruht (9) (Abb.2).

\section{Magnetresonanztomographie}

Die Sensitivität der MRI in der Pankreaskarzinomdiagnostik liegt nur unwesentlich über der Computertomographie und zeigt bzgl. der Resektabilitätsvorhersage im Bereich der V. mesenterica/V. portae, A. mesenterica superior ähnliche Werte wie die Computertomographie (75-81\%). Hauptvorteil der MRT-

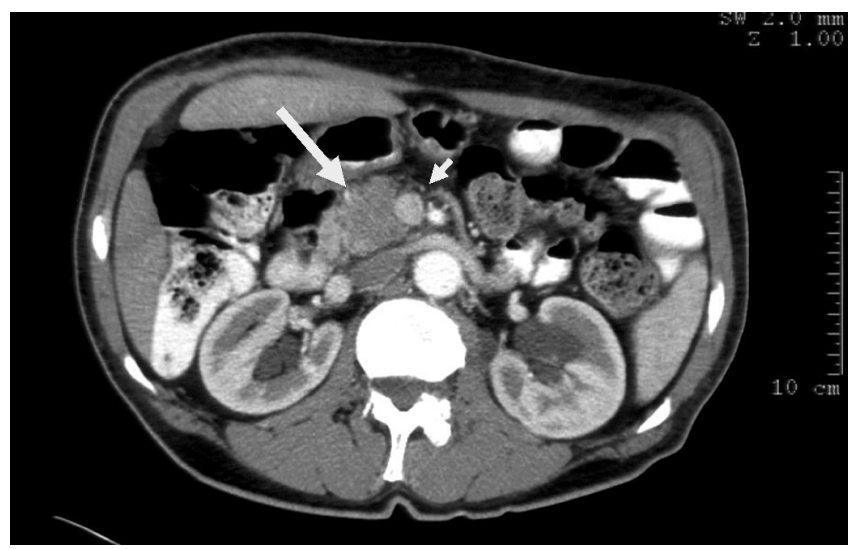

Abb.2 CT-Darstellung des Pankreaskopfkarzinoms des beschriebenen konreten Falls (S. 2325). (Leihgabe Abteilung für Radiodiagnostik Universitätskliniken Homburg). Pfeilmarkierung des Tumors (großer Pfeil), der keine sichere Trennschicht zur Venenachse zeigt (kleiner Pfeil).

Techniken liegt in der „All-in-One“-Diagnostik mit Kombination der MRT-Schnittbilddiagnostik, der MR-Cholangiopankreatographie (MRCP) und der MR-Angiographie (MRA) (3).

kurzgefasst: Die Computertomographie ist das Standardverfahren des Tumornachweises. Allerdings ist die Vorhersage der Resektabilität mit 60-70\% problematisch. Vorteil der Kernspintomographie ist die „All-in-One-Diagnostik“ mit MRCP und MRA.

\section{Endosonographie (EUS)}

Die geringe Vorhersagekraft von CT und MRI bzgl. der Resektabilität haben den Einsatz peripankreatisch einsetzbarer bildgebender Verfahren wie Endosonographie und laparoskopische Sonographie gefördert. In der Tat lassen sich durch beide Verfahren sowohl die Sensitivität als auch die Spezifität für die Diagnostik von Pankreasmalignomen auf $>80 \%$ bzw. 90\% erhöhen, wobei auch hier gilt, dass die Sensitivität beider diagnostischer Verfahren bzgl. einer tatsächlichen (histologisch verifizierten) Gefäßinfiltration unter $67 \%$ liegt $(2,4,11,20)$. Die erhöhte Sensitivität der endo- bzw. laparoskopisch sonographischen Verfahren ermöglicht die Diagnostik auch kleinerer Tumoren, somit früherer Tumorstadien, sowie die Beurteilung des regionalen Lymphknotenstatus. Die Spezifität der EUS kann durch eine Feinnadelbiopsie noch gesteigert werden (10). Diese darf transkutan wegen der Tumorstreuung entlang des Stichkanals nur mit äußerst strenger Indikationsstellung erfolgen.

\section{kurzgefasst: Der zusätzliche Einsatz der Endosonogra-} phie kann die Sensitivität und Spezifität der Diagnostik auf über $80 \%$ erhöhen, durch zusätzliche Feinnadelbiopsie kann die Spezifität erhöht werden. Umgebungsinfiltration und regionaler Lymphknotenstatus lassen sich mittels EUS am besten vorhersagen.

\section{Endoskopisch retrograde Cholangio-Pankreatikographie (ERCP)}

Da die überwiegende Anzahl der Pankreaskarzinome duktaler Natur sind, kommt der Pankreasgangdarstellung mit dem charakteristischen Befund des „Double-duct-sign“ (gleichzeitige 


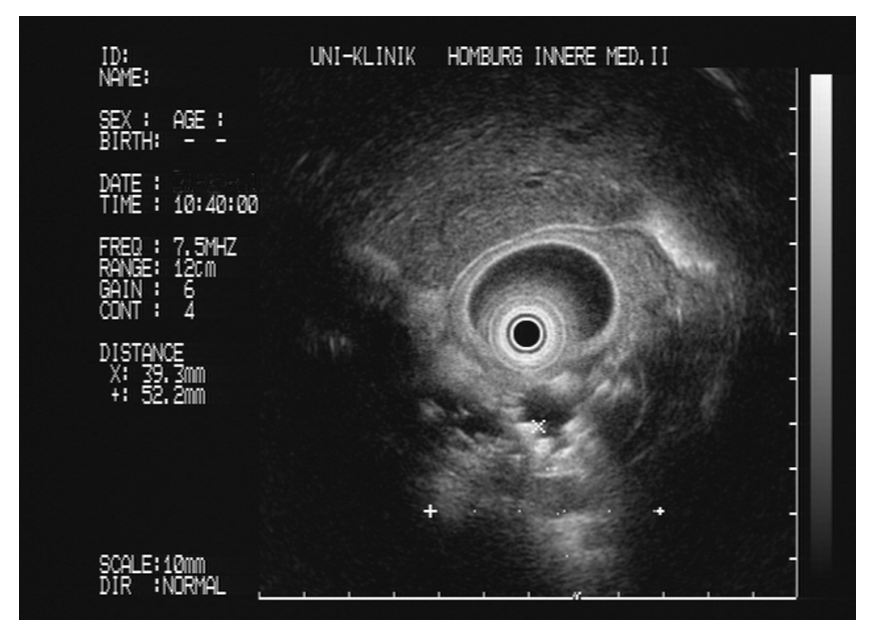

Abb.3 Endosonographiebefund eines Pankreaskopfkarzinoms (Leihgabe II. Medizinische Klinik Universitätskliniken Homburg). Tumor markiert $(x,+)$.

Stenose von Ductus pancreaticus und Ductus choledochus) nach wie vor ein hoher Stellenwert zu (18) (Abb.4). So bietet die ERCP im Vergleich zur MRCP den Vorteil der Intervention in Form der Gallengangsdrainage oder aber der zusätzlichen Gewinnung von zytologischem oder histologischem Material zur Diagnosesicherung. Erkauft wird dieser Vorteil durch eine im Vergleich zur MRCP gelegentlich relevante Morbidität in Form von Post-ERCP-Pankreatitiden oder Cholangitiden welche nachfolgende Operationen gelegentlich erschweren, oder aber Papillotomie-bedingte Komplikationen.

Neuere molekularbiologische Ansätze zeigen, dass durch den Nachweis von Mutationen im Bereich des Ki-ras-Onkogens im mittles ERCP gewonnenen Pankreassekret oder Bürstenmaterial die Sensitivität der Diagnostik gegenüber der konventionellen Zytologie deutlich gesteigert werden kann (24).

Die präoperative Stenteinlage zur Entlastung des Gallengangs wird kontrovers diskutiert. Während sie bei längerem Intervall bis zur operativen Sanierung (Resektion oder Bypass) z.B. infolge einer neoadjuvanten Therapie indiziert ist, kann bei kurzfristig anstehender Operation in der Regel auf die Stenteinlage verzichtet werden (2).

\section{kurzgefasst: Das „Double-duct-sign“ in der ERCP ist fast beweisend für ein Pankreaskarzinom. Der Vorteil der ERCP gegenüber allen anderen Verfahren liegt in der Möglichkeit zur Intervention sowie zytologischer, histo- logischer oder molekularbiologischer Diagnostik durch Pankreassekret oder Bürstenmaterial.}

\section{Angiographie}

Aufgrund der zunehmenden Verbesserung der CT-Diagnostik und Magnet-Resonanz-Tomographie mit der Möglichkeit der MRA hat die Angiographie im Rahmen des präoperativen Stagings an Bedeutung verloren. Sie ist in Einzelfällen zum Ausschluss von Gefäßvarianten oder dem präoperativen Nachweis einer relevanten Truncus-coeliacus-Stenose respektive Verschluss zur Operationsplanung sinnvoll und notwendig (7).

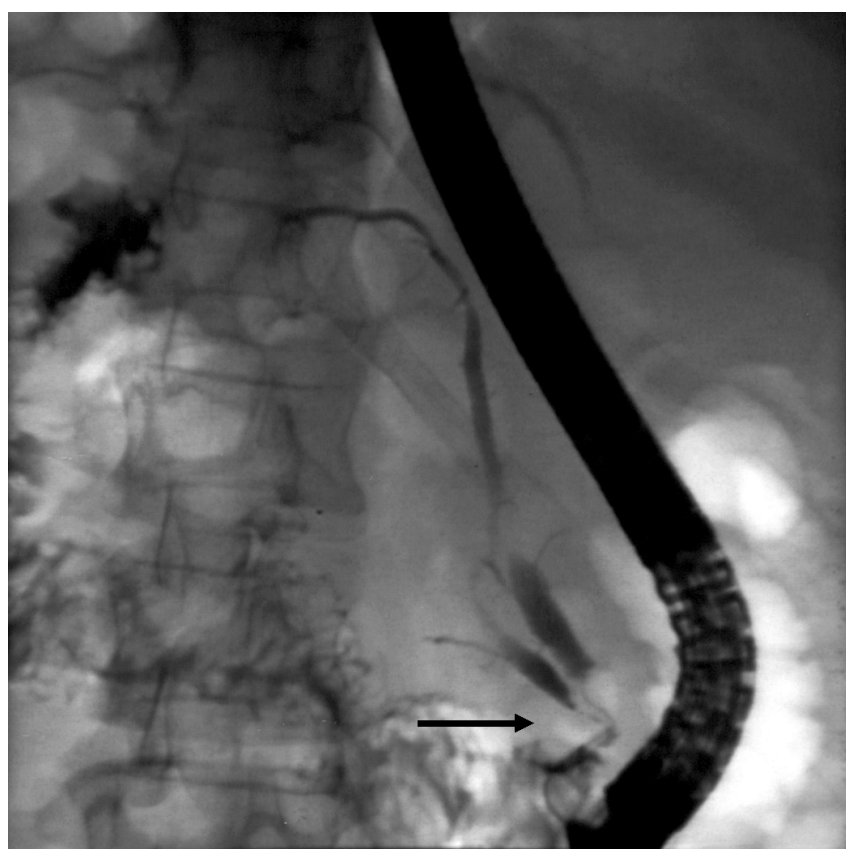

Abb.4 ERCP mit „Double-duct-sign“ bei Pankreaskopfkarzinom („Der konkrete Fall“)(Leihgabe II. Medizinische Klinik Universitätskliniken Homburg). Pfeilmarkierung Stenose Gallengang und Pankreasgang.

\section{Laparoskopie}

Ein beträchtlicher Anteil der Patienten mit Pankreaskarzinomen hat zum Zeitpunkt der Diagnosestellung bereits Lebermetastasen oder eine Peritonealkarzinose, so dass eine Resektion nicht mit kurativem Ansatz durchgeführt werden kann. Da bildgebende Verfahren kleine Lebermetastasen nur mit einer Sensitivität von ca. $20 \%$ und die Infiltration der Gefäße in $50-60 \%$ detektieren, wurde versucht mittels Laparoskopie die Vorhersage der Resektabilität bzw. Nicht-Resektabilität zu verbessern.

In einer vergleichenden Untersuchung fanden John und Mitarbeiter (15) für eine laparoskopisch gestützte Ultraschalluntersuchung einen positiven prädiktiven Wert (PPV) für die NichtResektabilität von 0,97, der PPV für US, CT und Angiographie lag bei 0,8. Conlon und Mitarbeiter gaben den PPV einer Laparoskopie für die Resektabilität mit 1 an (5). Launois wies in einer Übersicht jedoch darauf hin, dass bei den klinisch vermuteten Gefäßinfiltrationen letztendlich nur in ca. 50\% auch histologisch eine Infiltration gefunden wird. Es gibt derzeit keine diagnostische Modalität, die zwischen tumoröser Infiltration und peritumoraler desmoplastischer Reaktion unterscheiden kann (16). Auch für die Infiltration ins Dünndarmmesenterium, die dorsale Infiltration sowie kleine intrahepatische Metastasen können weder bildgebende Verfahren noch die Laparoskopie die manuelle Palpation des Befundes ersetzen.

\section{Tumormarker}

Problematisch ist die geringe Sensitivität und die eingeschränkte Tumorspezifität der Marker. CA19-9 hat die höchste Sensitivität (70\%) und Spezifität (87\%) und hat in erster Linie Bedeutung für die Rezidiverkennung im Follow-up $(3,13)$. 


\section{Weitere Verfahren}

Positronen-Emissions-Tomographie: Durch die PositronenEmissions-Tomographie kann neben der lokalen und Fernmetastasierung in Einzelfällen auch zwischen chronischer Pankreatitis und Pankreaskarzinom differenziert werden (12).

Intraduktale Sonographie und Pankreatikoskopie: Für diese neuen diagnostischen Verfahren liegen zum jetzigen Zeitpunkt keine Daten vor, die eine Bewertung zur Frühdiagnostik von Pankreaskarzinomen zulassen. Inwieweit hierdurch Fortschritte in der Beurteilung von kleinen malignen Strukturen im Pankreas bzw. Gallengang möglich sind, muss erst durch größere Studien belegt werden.

Nachsorgeuntersuchungen: Aufgrund der eingeschränkten Therapiemöglichkeiten erscheinen routinemäßige Nachuntersuchungen nicht gerechtfertigt. In der Regel erfolgt die symptomorientierte Diagnostik zur Planung der Palliation. Nach palliativer Stenteinlage wird wegen der Stentokklusion und der konsekutiven Probleme von einigen Autoren die dreimonatige Kontrolle empfohlen (21).

\section{Fazit fürr die Praxis}

Prinzipiell soll bei jedem Patienten über 60 Jahre mit neu aufgetretenem Diabetes und uncharakteristischen Oberbauchoder gar Rückenschmerzen durch eine Sonographie und im Zweifelsfall durch ein Kontrast-CT des Oberbauchs ein Pankreaskarzinom ausgeschlossen werden. Weiterführende Diagnostik ist nur bei begründetem Verdacht oder konkretem Tumornachweis zur weiteren Therapieplanung indiziert.

Autorenerklärung: Die Autoren erklären, dass sie keine finanziellen Verbindungen mit einer Firma haben, deren Produkt in dem Artikel eine wichtige Rolle spielt (oder mit einer Firma, die ein Konkurrenzprodukt vertreibt).
Literatur

1 Beger HG, Büchler MW, Friess H. Chirurgische Ergebnisse und Indikation zu adjuvanten Massnahmen beim Pankreaskarzinom. Chirurg 1994; 65: 246-252

2 Bissig KD, Mayerle J, Lerch MM, Friess H, Büchler MW. Der unklare Pankreastumor: Wann stenten, wann operieren? Dtsch Med Wochenschr 2002; 126 (Suppl 2); 107-114

3 Böhmig M, Wiedenmann B, Rosewicz S. Diagnostik und Staging des Pankreaskarzinoms. Dtsch Med Wochenschr 2001; 126: 113-116

4 Calett G, Ferrari A. Endoscopic ultrasonography. Endoscopy 1996; 28 : 156-173

5 Conlon KC, Klimstra DS, Brennan MF. Longterm survival after curative resection for pancreatic ductal adenocarcinoma: Clinicopathologic analysis for 5-year survivors. Ann Surg 1996; 223: 272-279

6 Doll R, Peto R, Wheatley K et al. Mortality in relation to smoking. 40 years' observation on male british doctors. Brit Med J 1994; 309: 901-911

7 Dooley WC, Cameron JL, Pitt HA, Lillemoe KD, Yue NC, Venbrux AC. Is preoperative angiography useful in patients with periampullary tumors. Ann Surg 1990; 211: 649-654

8 Falk RT, Pickle LW, Fontham ET. Occupation and pancreatic cancer risk in Louisiana. Am J Intern Med 1990; 18: 565-576

9 Freeny PC. Computed tomography in the diagnosis and staging of cholangiocarcinoma and pancreatic carcinoma. Ann Oncol 1999; 10 (Suppl); 12-17

10 Giovannini M, Seitz JF, Monges G, Perrier H, Rabbia I. Fine-needle aspiration cytology guided by endoscopic ultrasonography: results in 141 patients. Endoscopy 1995; 27: 171-177

11 Gress FG, Hawes RH, Savides TJ et al. Role of EUS in the preoperative staging of pancreatic cancer: a large single-center experience. Gastroint Endosc 1999; 50: 786-791

12 Ho CL, Dehdashti F, Griffeth LK, Busse PE, Balfe DM, Siegel BA. FDGPET evaluation of indeterminate pancreatic masses. J Comput Assist Tomogr 1996; 20: 363-369

13 Hollerbach S, Reiser M, Schmiegel HW. Die Pankreasraumforderung: Diagnostik und endoskopische Therapiemöglichkeiten. Dtsch Med Wochenschr 2001; 126 (Suppl 2); 126-135

14 Howe GR, Jain M, Burch JD et al. Cigarette smoking and cancer of the pancreas: evidence from a population-based-control-study in Toronto Canada. Int J Cancer 1991; 47: 323-328

15 John TG, Wright A, Allan PL et al. Laparoscopy with laparoscopic ultrasonography in the TMN staging of pancreatic carcinoma. World J Surg 1999; 23: 870-881

${ }^{16}$ Launois B, Stasik C, Bardaxoglou E et al. Who benefits from portal vein resection during pancreaticoduodenectomy for pancreatic cancer. World J Surg 1999; 23: 926-929

17 Lowenfels AB, Maisonneuve P, Cavallini G et al. Pancreatitis and the risk of pancreatic cancer. N Engl J Med 1993; 328: 1433-1437

18 Menges M, Lerch MM, Zeitz M. The double duct sign in patients with malignant and benign pancreatic lesions. Gastroint Endosc 2000; 52: 74-77

19 National Cancer Institute Bethesda. Annual Cancer Statistics Review 1973-1988. NIH publication No 91-2789, 1991

20 Rösch T. Staging of pancreatic cancer: Analysis of literature results. Gastrointest Endosc Clin N Am 1995; 5: 735-739

21 Schulz HJ, Braunschweig U, Schmidt H. Endoskopische Therapie der Gallengangsstenose. Dtsch Med Wochenschr 2001; 126 (Suppl 2); 136-140

22 Silverman DT, Brown LM, Hoover RN et al. Alcohol and pancreatic cancer in blacks and whites in the United States. Cancer Res 1995; 55 : 4899-4905

23 Smits NJ, Reeders JW. Imaging and staging of biliopancreatic malignancy: Role of ultrasound. Ann Oncol 1999; 10 (Suppl); 12-17

24 Van Laethaem JL, Vertongen P, Deviere J et al. Detection of c-Ki-ras gene codon 12 mutations from pancreatic duct brushings in the diagnosis of pancreatic tumours. Gut 1995; 36: 781-787

25 van Rees BP, Tascilar M, Hruban RH, Giardiello FM, Tersmette AC, Offerhaus GJA. Remote partial gastrectomy as a risk factor for pancreatic cancer: potential for preventive strategies. Ann Oncol 1999; 10 (Suppl); 204-207

26 Yeo CJ, Cameron JL, Sohn TA et al. Six hundred fifty consecutive pancreaticoduodenectomies in the 1990s: pathology, complications, and outcomes. Ann Surg 1997; 226: 248-257 